\title{
The Cloud Management System Based on Micro Station Cell Energy Management
}

\author{
Liu Hongyan \\ Beijing Information Technology College \\ 1241420132@qq.com \\ Liu Zhenyu \\ National Center For the Perform Arts \\ 112858658@qq.com
}

\begin{abstract}
In cellular networks, as a result of the heterogeneous network layout, a number of community, in the entire network, micro base station district energy management issues that need to be unified management and scheduling, and combined with the flow of communication and transport business and the efficiency of energy management, the development of energy management system based on cloud computing platform, according to the system, can have better energy efficiency, realize the communication and the advantage of energy balance. This article mainly from the two aspects of theory and actual implementation of this management system research.
\end{abstract}

Keywords: Cellular base station; cloud computing platform; energy management; energy scheduling

\section{Introduction}

Cellular communication technology developing rapidly in recent years, with the increase of the number of users, the increasing business volume, the amount of information transfer area information also increases exponentially, but the attendant problem is high energy consumption ${ }^{[1-2]}$. At present, the "green" has become a widespread concern of the whole society and advocate the theme, is directly related to the blue sky, pure air quality and health. With the popularity of smart mobile terminal, the rapid growth of various high-speed data applications, the communications industry energy consumption is increasing at an alarming speed. According to reports, including cellular network, energy consumption of the entire information communication industry accounted for $2 \%$ of global carbon dioxide emissions ${ }^{[3-4]}$. Energy consumption of mobile operators is highest in information communication industry and mobile network, energy consumption is growing faster than the industry average. With the rapid development of mobile communication technology, such as the widespread deployment of $3 \mathrm{G}$ and $4 \mathrm{G}$ system, will consume more energy. Research on mobile communication system, in the past only pay attention to the pursuit of system capacity and spectrum efficiency, with the global environmental situation is increasingly serious, urgent need to change to improve energy efficiency and balance of energy efficiency (EE) and spectral efficiency (SE) as the main target. From the operator's point of view, energy efficiency has great ecological and social responsibility in addressing climate change, also has significant economic benefits. According to statistics, the wireless base station (BS) energy consumption has accounted for nearly $60 \%$ of the mobile communication, as shown in Figure $1^{[5]}$. The huge energy consumption of the wireless base station also produced a large number of electricity, in mature markets in Europe, about $18 \%$ mobile operators operating expenses for electricity, 
while in developing countries India accounted for at least 32\%. Therefore, the energy saving effect is more direct and practical significance to conduct the research on energy efficiency of the base station side. Study on the effectiveness of energy if only to improve energy efficiency, reduce energy consumption, is bound to affect other communication system performance such as spectral efficiency (the frequency effect), delay, throughput and cost improvement of base station deployment. Therefore, as a key problem of scientific research personnel, energy efficiency and spectral efficiency is often not improved at the same time, improve the party and the other party will be reduced, which has become the bottleneck restricting the rapid development of the communications industry and energy saving.

From another point of view, the distribution in the mobile communication base station of different geographical areas need to be connected to the power grid, the power grid to provide energy. In recent years, smart grid as a new trend of the reform of the energy sector, is gradually in-depth research and pilot construction. In the smart grid, interactive features for the user to power system is an integral part of the operation and management, and encourage users to participate in power system helps to balance the supply and demand to ensure system reliability. The user to adjust the consumption time according to their demand for the ability to balance between power system and meet the power demand and consumption, can reduce or transfer the peak power demand of the power company to reduce operating expenses, to obtain environmental benefits by reducing the power loss and load units operation. Because of the energy storage technology is backward, the power supply and demand as a special commodity with moment balance, namely production must be consumed immediately. Price based demand side response including the TOU price, spot price (RTP) and peak electricity price, the implementation of real-time electricity price to guide the user consumption of power resources is an important part of the construction of smart grid. Based on real-time price guide encourage users to reduce the peak load and load is low in power consumption, the power grid safe operation at high load rate, achieve the purpose of peak load shifting, so as to improve the rate and power efficiency of power equipment. Therefore, strengthening the cooperation of mobile communication base station provides smart grid energy and energy demand, has very important practical significance. The power of smart grid as a special energy capture technology, relying on base station power supply of smart grid, to adjust energy consumption according to their real-time electricity price. Because of the diversification of energy in smart grid, including new energy, traditional energy volatile, the total social energy consumption is constantly changing, energy consumption can only rely on price means to adjust the user to ensure the balance of power of the moment. The price of electricity can save energy by more low for other resources or low efficiency for other performance improvements, such as spectrum efficiency, delay, bandwidth and power; the price is high, is to reduce energy consumption and improve energy efficiency as the main target. 


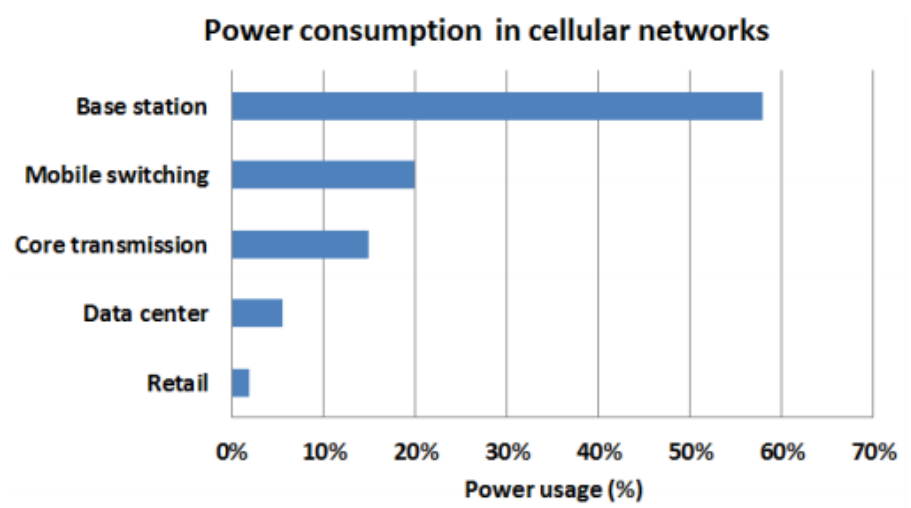

Figure 1. Cellular Network Power Consumption Ratio

The next generation of communication will be widely used in cognitive and heterogeneous technologies, can be seen from the Shannon formula, and the bandwidth capacity is proportional, and signal power and capacity is in direct proportion to the logarithm of the relationship. In the same capacity requirements, signal power to more than the cost of spectrum pay, efficient use of spectrum resources is also visible for energy saving. The use of cognitive technology, macrocell microcell make full use of idle spectrum perception to the formation of the available spectrum pool, the spectrum of available opportunities, or it can be used to increase the spectrum resources, more spectrum cost to improve system capacity; The spot price of smart grid (including renewable energy supply), the increased availability of energy, or the existence of excess energy resources, so we can improve the system capacity by using more energy cost. Therefore, increasing the spectrum and energy of the two dimensions of opportunity balanced energy efficiency and frequency effect may provide, increased design freedom, the abundance of different resources decided to use what kind of resources to meet the system performance requirements.

The heterogeneous layout through the micro cellular technology, providing high-speed service but also reduces energy consumption, in line with the green network design trends from system structure. The transmission of energy efficiency from the angle of resource management, can understand at the right time and appropriate allocation of resources will transmit the information to the right user. The effective management of time domain, frequency domain and the airspace resources involved, can be carried out from time scale. As a long time scale including new bandwidth resource acquisition, flexible hardware design, micro cellular deployment and other measures; in time scales including cellular switch, cell radius zoom and other measures; short time scales including resource allocation, adaptive bandwidth adjustment, discontinuous transmission technology, cellular MIMO suppression technology measures. Implementation of multi domain resource management and physical layer transmission design helps heterogeneous wireless network efficiency and frequency equalization target.

However, in the optimization of the energy of the physical layer and other layers is limited, can make the control more accurate, requires a lot of network data, the joint control in a wider scope. Therefore, this requires scheduling method of micro cellular base station for cloud computing, considering the comprehensive quality of communication, real-time pricing and other elements, so we construct the scheduling of cloud platform is very important, has academic and practical value and significance. 


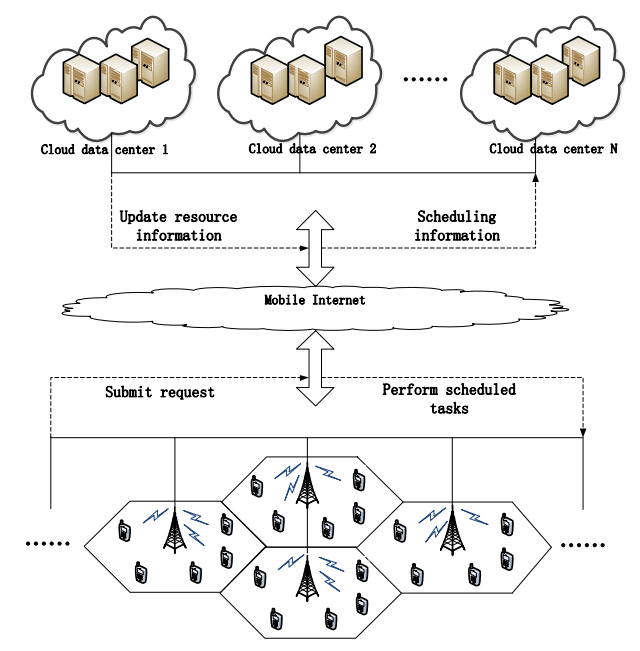

Figure 2. Mobile Internet Topology Map

As shown in Figure 2, by each base station, including the micro base station and Acer station, the power and the real-time electricity price to the mobile Internet through the link, transfer to the mobile Internet data center, the data center according to the historical data and related influencing factors, distribution power of each base station. Previous studies mainly focus on the physical layer and data link layer through a small range of power dispatching.

\section{Related Works}

\subsection{Research Status of Green Communication}

Green wireless communication is a comprehensive interdisciplinary research, direction, energy conservation, resource conservation, Protect environment has become a major front topic in the field of wireless communication, in recent years by the University of western countries, scientific research institutions and large enterprises more and more attention. The international standard organization such as 3GPP and ITU have issued some relevant draft, some of the energy efficiency of wireless communication in international research projects have been carried out, such as Green Radio, the EU's seventh framework program in the EARTH (EnergyAware Radio and neTwork tecHnologies) and OPERANet (Optimizing Power Efficiency in mobile RAdio NETworks). Some valuable papers have been published on the topic, green communication is also ICC, GLOBECOM and PIMRC and other well-known international conference organization, In recent years, the focus gradually shifted to the energy efficiency of the consideration of the reality, the different scenarios of law, energy efficiency and spectral efficiency equilibrium of the hotspot, and began to study energy-saving has been focused on the base station side. Therefore, the green energy efficiency in wireless communication is gaining more and more attention in the world, the relevant academic activities more and more active. Such is the first subject of wireless network node power supply is limited, such as the node of wireless sensor network MAC layer design, network routing protocol design and Research on energy saving, energy saving of mobile communication terminal; But with the development of mobile communication network, mobile communication base station energy consumption accounted for the increase, began to strengthen the study of [6] on the energy consumption of the base station side. Summarize the relevant literature, research content involved mainly includes five aspects as follows. In order to evaluate the effects of 
different system components on the effectiveness of wireless network energy, a general definition of the energy consumption model to provide accurate estimation of energy consumption. The model of energy consumption model is not only energy consumption status, and analysis of the overall cost of ownership of cellular network, describe the main cost expenditure and energy consumption, including operating expenses and capital expenditure. At present, the main energy consumption model is to evaluate the economic effects of different energy saving solutions or ecological effects, taking into account the practical considerations. Effect of two kinds of different components of the access network model to assess the effect on energy consumption, and considering the effects of different types of base station, radio frequency structure and backbone network. The definition of green degree for the measurement and criterion of energy efficiency, provide status information of different components of the energy consumption of direct comparison and evaluation of the network. Energy efficiency criterion in the protocol layer design and optimization model is directly related to the judge, has play a decisive role role. The relevant standards include European Technical Standards Association and the telecommunications industry solutions alliance, organization, research institutions, have to participate in the formulation of energy efficiency criterion. The overall energy efficiency criterion can be divided into three categories: element level, equipment level and the level. Element level criterion is main measure specific device in a wireless device (such as antenna, power amplifier and so on) performance, such as PUE (Power Usage Efficiency); EE performance level evaluation criterion of main equipment of single equipment, such as TEEER (Telecommunications Equipment Energy Efficiency Rating) and ECR (Energy Consumption Rating) [17] network level criterion and describe network; the performance of energy consumption and the measurement equipment (such as network capacity and coverage).

From the energy consumption of base station in a cellular network energy consumption in the proportion can see, reduce energy consumption and base station itself is a very important measure. The existing measures include improving the base station power amplifier energy consumption, reduce the energy consumption of the base station to develop protocols such as depending on the size of the load off the base transceiver station etc.; Base station power management strategy for energy aware, can be adjusted according to load power station to adjust the service radius, and cooperation with neighboring base stations, considering load conversion RF frequency planning, threshold setting, to avoid coverage holes, space and time on the ups and downs of the tracking in practical design. In addition, reduce the number of base stations and the use of renewable energy is also some effective measures, such as the Nokia Siemens network Flexi radio base station. Requirement of higher data rate growth requires more intensive deployment of base stations in a cellular network, the macrocell is mainly designed to provide a wide range of coverage and not high data rate and reduce energy consumption, and change the existing network structure is not economical. There is a more feasible method to shorten the transmission distance so as to reduce power and improve the transmission rate, cellular network deployment scheme based on small micro cellular, cellular picocell and femtocell design is very promising. However, coverage, performance prediction, interference and mobility management and security issues are in the design of this kind of problem to solve the heterogeneous network.

\subsection{Research on the Construction Status of Cloud Platform}

Since the birth of cloud computing technology, there has been no definition is very accurate, so the term cloud computing can be used to describe many at the same time with "common cloud" platform or system. The application characteristics and the 
characteristics of the present platform of cloud computing division, cloud computing technology is mainly divided into three categories ${ }^{[7-9]}$ : infrastructure-as-a-service (Infrastructure as a Service, laaS), platform as a service (Platform as a Service, PaaS) and software as a service (Software as a Service, SaaS). The relationship between laaS cloud computing platform is constructed based PaaS cloud computing platform, PaaS cloud computing platform is the basic structure of SaaS cloud computing platform. LaaS cloud computing platform to provide users of all server infrastructure (including CPU, memory, disk and network) or rental service. The user can run the infrastructure operating system or application software based on. Users only need to apply for and manage the virtual infrastructure resources, without considering how the hardware maintenance. PaaS cloud computing platform to provide users can meet the configuration requirements, development platform for the user, for the user to complete their required work. The PaaS cloud computing platform shielding users for hardware, infrastructure, and even the maintenance of the operating system, users only need to spend their energy in how to use cloud computing services to provide the platform to complete the work.

SaaS cloud computing platform to provide a variety of software for the user, allowing users to select one or several to meet the needs of users. The SaaS cloud computing platform and PaaS cloud computing platform, it provides users with a single software, users only need to focus on how to use the software service for users, saves the hardware, operating system and application configuration installation work. LaaS cloud computing platform virtualization technology based on the management of physical server resources, providing infrastructure resources needed to the user in the form of a pool of resources, the realization of the physical server and application deployment of loose coupling. At present, laaS cloud computing platform mainly through to provide infrastructure resources to provide users with a virtual machine, in the Green Computing Initiative, and the source of the laaS cloud computing platform like the spring rain, many of them are not open-source platform used by many users even know, currently in the domestic community with high activity and widely used are OpenStack and Eucalyptus and other cloud computing platform.

OpenStack is aimed at the United States National Aeronautics and Space Administration in cooperation with Rackspace and the software of open source projects for the construction and management of public and private cloud. OpenStack cloud computing platform mainly consists of two independent parts: Nova and Swift, where Nova is the America National Aeronautics and space administration to develop a virtual server deployment and business computing module, Rackspace module and Swift is distributed cloud development, both of them can be used separately, but also can be used in conjunction with. As the largest community, participate in the largest number of open-source cloud computing platform OpenStack, not only that, OpenStack also received technical support and investment of hundreds of large international companies. OpenStack is an open source laaS cloud computing platform, fully realized by the python script. OpenStack from the function division, mainly Nova Compute, Glance and Swift. Nova Compute is mainly responsible for the virtual machine management function, the Glance module is mainly responsible for the image management function, and the Swift module is mainly responsible for the large capacity storage to provide high availability. The three asynchronous communication by MessageQueue, low degree of coupling of the platform to improve robustness and scalability.

The Nova component of OpenStack is playing the role of the controller, mainly responsible for the management of virtual machine instance life cycle, at the same time can be set up special network for a specific project has multiple instances. OpenStackNova provides comparable to Amazon EC2 in the public cloud resources, and in the private cloud favorably in most commercial cloud products. In the public cloud, 
providing object storage mechanism for a user to the virtual machine image storage prefabricated, a user can start to mirror for virtual machine templates.

\section{Proposed Scheme}

This paper developed a cellular base station energy management system based on cloud platform, this system can be based on the data feature of energy management, and the characteristics of the data transmission, the energy distribution of cloud platform and cloud data to predict the software design, but for a cloud platform of communication and information transmission, this paper go, so the only software development for the data center in detail.

\subsection{Implementation of Power Allocation Based on Software}

We can see from the architecture that based on the cloud platform intelligent library management system consists of 6 layers and 2 service modules, where in the 6 layer respectively, data source, data integration, data storage, data analysis and processing, information display and business applications, 2 service modules for the entire system to provide security service and access control service.

\section{1) Data Source}

The system supports multiple types of data source access, mainly include two categories: The first category is the intelligent management of the relevant business data, related systems include a library login data, the user's browser to borrow the relevant data acquisition system, purchasing system data classification system data and presents; the second is external data, including data such as book information providers, system access to such data from authoritative data distribution mechanism.

2) Data Integration

This layer uses parallel ETL technology loading the data of the data source layer by extraction, transformation, loading and a series of processing to the data storage layer. For different types of data sources we offer different ETL interfaces, and the degree of parallelism ETL process can be set as desired.

3) Data Storage

The Data of this system storage in the cloud data warehouse, data warehouse uses a distributed cloud storage technology to meet the massive data storage requirements, not only safe and reliable, but also economical and easy to expand. In order to meet the user's real-time and non-real-time data processing needs, cloud data warehouse is divided into two parts: ODS and distributed data warehouse, where the ODS can meet the needs of real-time data storage, distributed data warehouse to meet the non-real-time data storage requirements. To meet the specific needs of data exchange between the ODS and the distributed data warehouse, this system uses interfaces

4) Data Analysis and Processing

Data analysis and processing layer for the data in cloud warehouse provides three types of processing: ad-hoc queries, multidimensional OLAP analysis, data mining and machine learning. Ad hoc queries can meet user's demand for real-time data query and these data are stored in the ODS part of data storage layer; Multidimensional OLAP analysis provides multi-dimensional analysis based on parallel computing technology to meet user's demand for OLAP analysis of massive data; Data mining and machine learning provides clustering, forecasting, outlier analysis, correlation analysis and other types of parallel data mining algorithms, supporting users to mine the unknown, valuable knowledge from vast amounts of data, thus providing a basis for decision making by managers.

5) Information Display 
Information presentation layer mainly adopts simple appearance, landscaping tools FusionCharts as the display mode, dynamicly displaying index and parameter, including histogram, pie chart, line chart, real-time map, scale diagram and other forms of chart .

6) Business Applications

Business application layer provides multiple levels of applications for smart electricity, including the amount of fees analysis, borrow books analysis, recommended literature analysis, user behavior analysis and so on.

7) Service Module

System service module provides security and access control services for the system. Security should defend application security, authentication mechanism, application security auditing, data storage confidentiality, data storage integrity, non-repudiation, software fault tolerance, resource control, backup and restore application data and other aspects of security; Access control services for system resources provides flexible rights allocation mechanism, while ensuring the safe use of system resources.

ETL layer should meet the following specifications:

$>$ (1)Support data extraction in HBase data tables provided from Oracle databases and WebService;

$>$ (2) Support the data written back from HBase to Oracle;

$>$ (3)Support ETL process parallelism, you can set the degree of parallelism by parameters;

$>$ (4)Support deltification ETL process, in accordance with a fixed field for continuous incremental data extraction, rather than each time the total amount of all data extraction;

> (5)Support HBase rowkey optimization, the date of conversion, a variety of data transformation rules string concatenation, substring and other character strings;

$>$ (6)Support discarding abnormal data, stop running, recording a variety of exception handling logs.

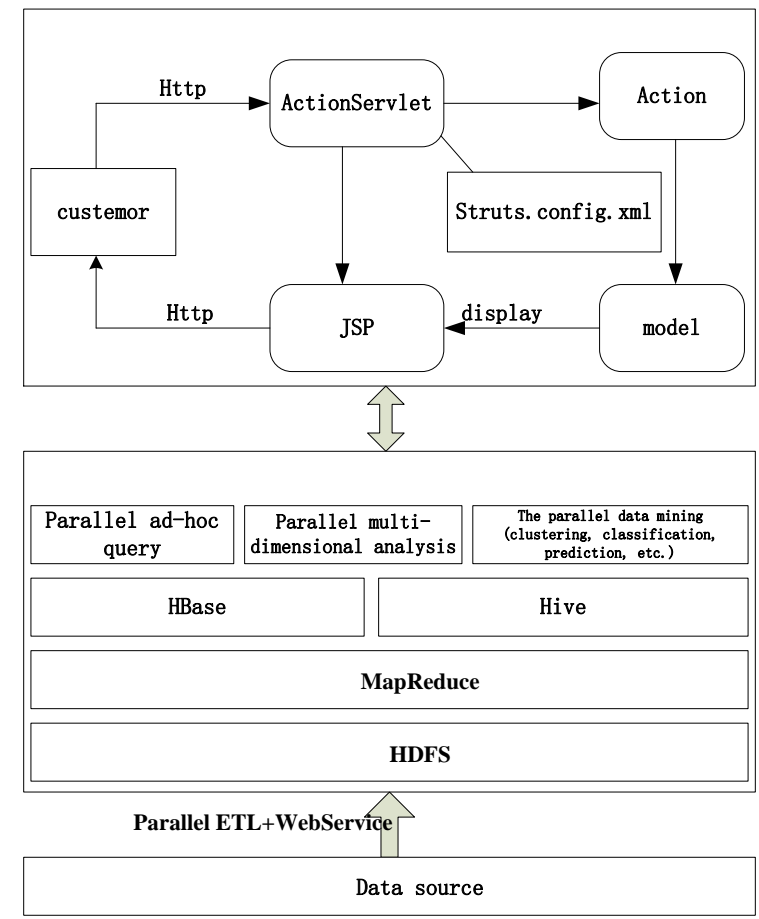

Figure 3. Cloud Computing Platform Architecture Diagram

We can see from Figure 3, the data source of XQBI system mainly stores in relational 
databases, or a third party providing through the WebService interface. Using parallel ETL technology and WebService technology,the system will integrat the data source into the cloud data warehouse. Cloud Data Warehouse through a distributed file system HDFS and HBase distributed database for storing large amounts of data while using MapReduce parallel computing framework can analyse and process efficiently. Analysis of the data processing system is based on parallel computing technology, including ad hoc queries, multidimensional analysis and data mining three levels, where the multi-dimensional analysis of class-based SQL Hive provide the interface, data mining include clustering, classification, prediction, and other type. Applications XQBI system implementation and the results show the use of a common J2EE architecture. XQBI in various parts of the system, whether it is the data source, the background, the foreground has security isolation technology and resource authority role-based control technology to provide them with reliable and complete security mechanism.

Data integration uses data of multiple heterogeneous data sources through the extraction, transformation, loading a series of operations in accordance with the unified format eventually centrally stored procedure. XQBI system needs to access different data sources, including multiple intelligent data cell home appliances, information collection system and marketing business data, weather data and other third party, according to the needs of different data sources can access different data integration technology, intelligent Community household appliances data, information collection system and marketing business data is typically stored in existing relational database systems, parallel to these data we use ETL technology for data integration; and for third parties, weather data, general specific Web Service interface provided by a third party to acquire, eventually consolidated by the data in accordance with business needs unified storage in the cloud data warehouse.

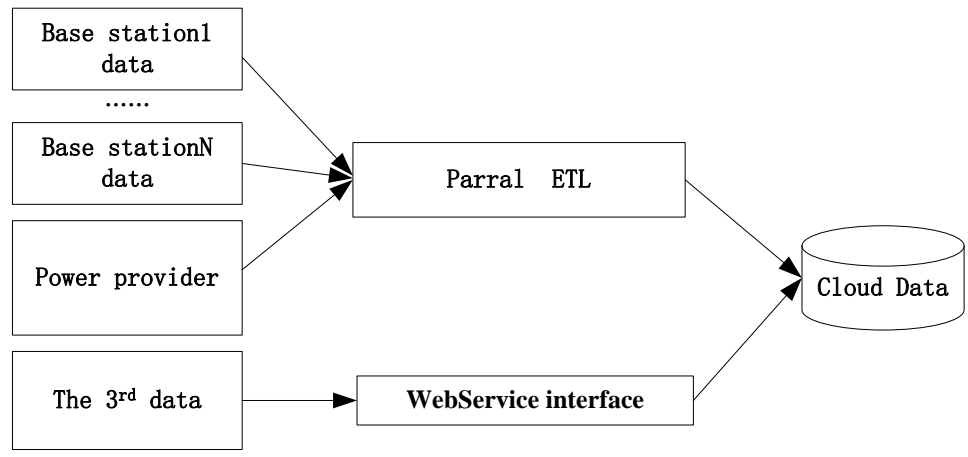

Figure 4. The Data Consolidation Process

\subsection{Forecast Based on Data}

Implementation can be divided into three steps:

First: Map Reduce Driver set the relevant parameters, including the number of Map and the number of Reduce is 1 ;

Second: Map stage, the use of KNN, chooses from the most recent forecast point $\mathrm{x} \mathrm{K}$ points in each block of data processed in Map;

Calculation the distance $\mathrm{D}$ between $\mathrm{x}$ and $\mathrm{x}_{\mathrm{i}}$ and choose five minimum distance points as the local forecast information points. These points will be recorded as $x_{1}, x_{2}, x_{3} \ldots x_{k}$. Third: Reduce stage, incluing four sub-phases:

(1):Compare the distance $\mathrm{K}$ points in each Map with $\mathrm{x}$, select the $\mathrm{K}$ smallest as KNN algorithm ultimately selected $\mathrm{K}$ points.

(2):Determine the weights. 
According to $\mathrm{K}$ points in the previous step, select the right distance calculations to predict the point $\mathrm{x}$ of weight. Weights calculated using Gaussian mixture model.

(3):Weighted linear regression.

(1) Assume $f(x)=\beta_{0}+\beta_{1} m+\beta_{2} h+\beta_{3} w+\beta_{4} t$, calculating the coefficient $\beta$ with the weighted point.

(2) According to the least squares method, the $\beta$ is required, which makes the equation $\sum_{j=1}^{k} w_{j}\left[y_{j}-f\left(x_{j}\right)\right]^{2} \quad$ minimum.

$$
\text { ( } 3 \text { ) Represent } \beta=\left(X^{T} W X\right)^{-1} X^{T} W Y \text { with matrix, in which }
$$$$
Y=\left[\begin{array}{llll}
y_{1} & y_{2} & \ldots & y_{\mathrm{k}}
\end{array}\right]^{T}, \beta=\left[\begin{array}{llll}
\beta_{0} & \beta_{1} & \ldots & \beta_{5}
\end{array}\right]^{T} \text {. }
$$

$$
X=\left[\begin{array}{cccccc}
1 & u_{1} & m_{1} & h_{1} & w_{1} & t_{1} \\
1 & u_{2} & m_{2} & h_{2} & w_{2} & t_{2} \\
\ldots & \ldots & \ldots & \ldots & \ldots & \ldots \\
1 & u_{k} & m_{k} & h_{k} & w_{k} & t_{k}
\end{array}\right] ; \quad W=\left[\begin{array}{llll}
w_{1} & & & \\
& w_{2} & & \\
& & \ldots & \\
& & & w_{k}
\end{array}\right]
$$

(4):Forecast. Put the information of $\mathrm{X}$ into the equation to calculate and get the predicted results.

\section{Results and Analyze}

In this paper, the case is depended on the calculation and simulation of the cloud computing model for the mobile Internet. So we need to build a cloud simulation platform. This cloud platform uses computing simulation model platform CloudSim, developing from its foundation by the distributed parallel computing on using this platform can store computer simulation data and transfer resources, but its lack of link topology changes, according to the realities of the present experiment this has been modified, the modification according to the topology diagram to modify its transmission and transmission of data time. Simulated environment, including computer configuration environment. Its computer simulation environment shown in Table 1:

Table 1. VM Configuration

\begin{tabular}{cccc}
\hline & Processor & Memory & Hard disk \\
\hline$V M_{1}$ & $1 \times 2$ & $4 \mathrm{GHz}$ & $500 \mathrm{~GB}$ \\
& $\mathrm{GHz}$ & & \\
$V M_{2}$ & $2 \times 2$ & $8 \mathrm{GHz}$ & $1 \mathrm{~TB}$ \\
& $\mathrm{GHz}$ & & \\
$V M_{3}$ & $4 \times 2$ & $16 \mathrm{GHz}$ & $2 \mathrm{~TB}$ \\
& $\mathrm{GHz}$ & & \\
$V M_{4}$ & $8 \times 2$ & $32 \mathrm{GHz}$ & $4 \mathrm{~TB}$ \\
\hline
\end{tabular}


The simulation process is generally shown below, according to the virtual task and scheduling implementation model for the development of its core algorithm written, its scheduling intervals depending on the simulation environment, the need for a separate set.

From the resource utilization of Figure 5 , we can see that average energy consumption of the proposed method is significantly lower than other programs, we can see that the proposed scheme can have a strong computing power in business; which can be fast data mining tourism information high, resulting in a mobile cloud platform tourist information advice efficiency. As can be seen, the computational efficiency of the proposed travel more mobile cloud platforms.

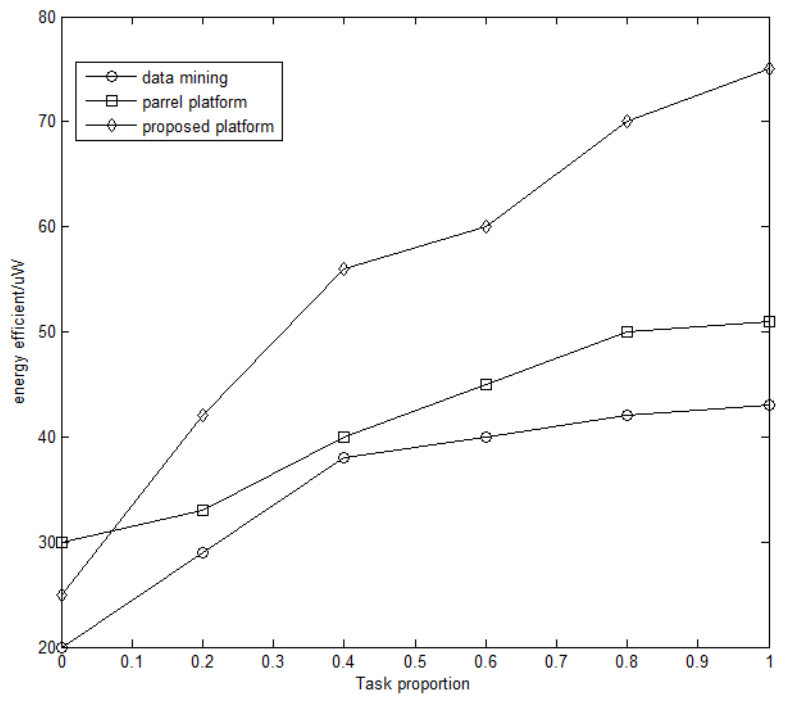

Figure 5. The Average Energy Consumption Contrast

As can be seen from Figure 6, the user information data mining and predictive accuracy of the data referred to herein is high, to better reflect the needs of users, providing users with the demand for their research needs and other aspects of to make more rational and scientific judgment provided favorable data support.

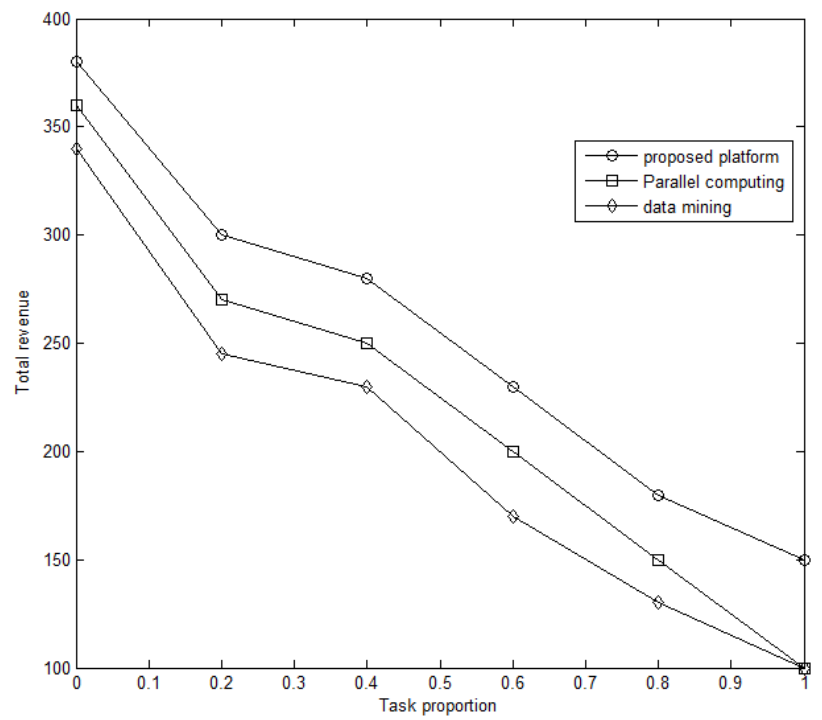

Figure 6. The Total Revenue Contrast 


\section{Conclusion}

Based on the characteristics of mobile Internet and information characteristics of library management, this article built filter mode data. In terms of computing resources allocation, we use the optimization of energy efficiency programs, taking the business process of time into account by experiment platform and the performance aspect of resource utilization has been greatly improved. This article also proposed data to predict user behavior, giving full play to the advantages of cloud platforms. Experimental results show that the proposed algorithm has a higher accuracy.

\section{References}

[1] W. Song, X. Su, "Review of mobile cloud computing[C]//Communication Software and Networks (ICCSN)", 2011 IEEE 3rd International Conference on. IEEE, (2011), pp. 1-4.

[2] H. Qi, A. Gani, "Research on mobile cloud computing: Review, trend and perspectives[C]"//, Digital Information and Communication Technology and it's Applications (DICTAP), 2012 Second International Conference on. ieee, (2012) pp. 195-202.

[3] L. Yang, J. Cao, Y. Yuan, "A framework for partitioning and execution of data stream applications in mobile cloud computing”. ACM SIGMETRICS Performance Evaluation Review, vol. 40, no. 4, (2013), pp. 23-32.

[4] N M Rao, C.Sasidhar, V S KumarCloud computing through mobile-learning[J]. arXiv preprint arXiv:1204.1594, (2012).

[5] Y. Cui, X. Ma, H. Wang, "A survey of energy efficient wireless transmission and modeling in mobile cloud computing", Mobile Networks and Applications, vol.18, no. 1, (2013), pp. 148-155.

[6] Y. Cui, X. Ma, H. Wang, "A survey of energy efficient wireless transmission and modeling in mobile cloud computing[J]”, Mobile Networks and Applications, vol.18, no. 1, (2013), pp. 148-155.

[7] Z. Zhao, K. Hwang, J. Villeta, "Game cloud design with virtualized CPU/GPU servers and initial performance results",//Proceedings of the 3rd workshop on Scientific Cloud Computing Date. ACM, (2012), pp. 23-30.

[8] Y. Cui, X. Ma, H. Wang, "A survey of energy efficient wireless transmission and modeling in mobile cloud computing[J]",Mobile Networks and Applications, (2013), vol. 18, no. 1, pp. 148-155.

[9] Z. Sanaei , S. Abolfazli, A. Gani, "Tripod of requirements in horizontal heterogeneous mobile cloud computing" arXiv preprint arXiv:1205.3247, (2012).

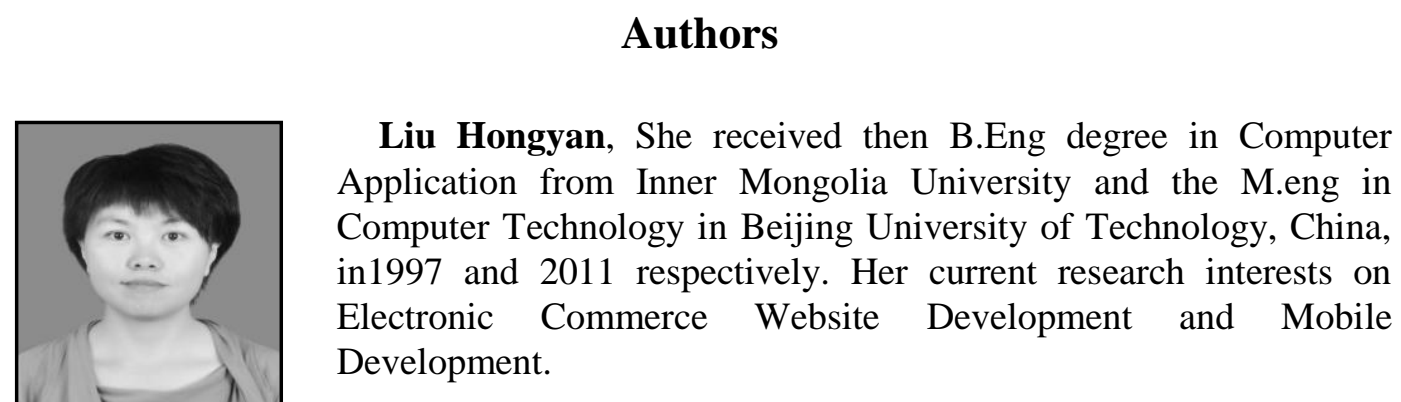

Liu Zhenyu, He received then B.Eng degree in Computer $(2-\infty)$ Application from Inner Mongolia University and the M.eng in Computer Technology in Beijing University of Technology, China, in1997 and 2011 respectively. His current research interests on Website Development and Network Security. 